\section{Análise dos registros de atendimentos de mulheres que realizaram aborto previsto em lei em Porto Alegre, Rio Grande do Sul, Brasil}

\author{
Analysis of records for women that have undergone \\ legally authorized abortions in Porto Alegre, \\ Rio Grande do Sul State, Brazil
}

\begin{abstract}
Análisis de los registros de atención a mujeres que abortaron según lo previsto en la ley en Porto Alegre, Río Grande do Sul, Brasil
\end{abstract}

\author{
Maura Carolina Belomé da Silva 1,2 \\ Daniela Riva Knauth 1 \\ Rogério Boff Borges 3 \\ Luciana Neves Nunes 1 \\ Camila Giugliani 1
}

\section{Resumo}

O aborto permitido por lei no Brasil é um direito pouco garantido devido a múltiplas barreiras de acesso. A dificuldade em obter-se registros confiáveis e de fácil acesso, que subsidiem ações de monitoramento e avaliação pertinentes a essa temática, é outra questão preocupante relacionada à organização do sistema de saúde. Assim, esta pesquisa teve como objetivo analisar os registros de atendimentos a mulheres que realizaram aborto legal no Municipio de Porto Alegre, Rio Grande do Sul, no período de 2013 a 2018 e identificar sua prevalência no Sistema de Informações Hospitalares (SIH). Para isso, os prontuários dos casos de aborto previstos em lei, realizados nos quatro serviços de referência foram avaliados in loco. Os dados obtidos foram cruzados com o banco do SIH do Sistema Único de Saúde (SUS). A razão de prevalência foi estimada por meio do modelo de regressão Poisson, com variância robusta. Foram identificados, nos prontuários, 236 casos de aborto legal, dos quais 95 (40,3\%) estavam registrados no SIH. Os casos cujos prontuários tinham o CID O04 (aborto por razões médicas) identificado em seus registros internos tiveram uma prevalência de 3,02 (IC95\%: 1,83-4,98) vezes de constarem no SIH do que aqueles que não identificaram. Foi observado que o número de registros de aborto legal no SIH diferia dependendo do hospital; no entanto, verificou-se que, independentemente do hospital, o fato do registro interno descrever o CID O04 aumenta a prevalência de registros no SIH. Conclui-se que a falta de padronização e o sub-registro dificultam a obtenção de informações fidedignas na base de dados nacionais do SUS, aumentando a invisibilidade do aborto legal.

Aborto Legal; Classificação Internacional de Doenças; Sistemas de Informação; Registros

\author{
Correspondência \\ M. C. B. Silva \\ Rua General Rondon 737, Porto Alegre, RS 91900-121, Brasil. \\ maurabelome@gmail.com \\ 1 Universidade Federal do Rio Grande do Sul, Porto Alegre, \\ Brasil. \\ 2 Secretaria Estadual da Saúde do Rio Grande do Sul, Porto \\ Alegre, Brasil. \\ 3 Hospital de Clínicas de Porto Alegre, Porto Alegre, Brasil.
}




\section{Introdução}

Pelo Código Penal Brasileiro 1, o ato de provocar um aborto é considerado crime, exceto em três circunstâncias: risco de vida da gestante, anencefalia do feto e gravidez resultante de violência sexual 2,3. Contudo, a previsão legal do direito ao aborto, nesses três casos, não é sinônimo de garantia de acesso. $\mathrm{O}$ direito de uma mulher que busca pelo aborto legal é frequentemente restringido por diversos fatores: escassez de serviços de referência; despreparo técnico das instituições; desarticulação da rede de atenção à saúde; desconhecimento da legislação por parte da sociedade em geral e até por parte dos profissionais; exigências desnecessárias e incorretas, como a apresentação do boletim de ocorrência; ou alegação de objeção de consciência por parte dos profissionais 4,5.

Entre 2013 e 2015, foram realizados apenas 2.442 abortos legais em todo Brasil, conforme pesquisa de representatividade nacional 6 . Por meio da análise de 1.283 prontuários, constatou-se que $94 \%$ dos casos eram situações de estupro. O mesmo estudo também relata que, dos 68 serviços habilitados existentes, apenas 37 haviam de fato realizado interrupções de gravidez decorrentes de estupro, e que $80 \%$ dos atendimentos em todo Brasil foram realizados por um único hospital. Os hospitais atuantes estavam distribuídos em 20 Unidades da Federação (cinco na Região Norte, 11 no Nordeste, três no Centro-oeste, seis no Sul e 12 no Sudeste), sendo que em sete estados não havia nenhum serviço ativo 6 . No Rio Grande do Sul, conforme dados de 2019, há sete serviços de referência para o atendimento do aborto previsto em lei: quatro em Porto Alegre, um em Canoas, um em Caxias do Sul e um em Rio Grande (Departamento de Informática do SUS. Sistema de Informações Hospitalares. http://www2. datasus.gov.br/DATASUS/index.php?area=0202\&id=11633, acessado em 02/Fev/2019).

Além da pouca disponibilidade de serviços e das demais barreiras de acesso já citadas, outra questão, relacionada à organização do sistema de saúde, mostra-se preocupante: a dificuldade na obtenção de registros confiáveis e de fácil acesso para a avaliação e o planejamento de ações voltadas a essa temática no Sistema Único de Saúde (SUS). No Brasil, o aborto legal é um procedimento hospitalar e, assim, deveria ser identificado com um número de procedimento específico no Sistema de Informações Hospitalares (SIH) do SUS 7. Contudo, atualmente, não existe um código de procedimento que identifique o aborto previsto em lei e gere um pagamento por ele. Em 21 de maio de 2014, o Ministério da Saúde emitiu a Portaria no 415 8, que criava o procedimento: "Interrupção da gestação/antecipação terapêutica do parto prevista em lei". Entretanto, essa portaria foi revogada oito dias após a sua publicação ${ }^{9}$. Hoje, é possível achar no SIH apenas a identificação da 10a revisão da Classificação Internacional de Doenças (CID-10): CID O04 - Aborto por razões médicas e legais quando o profissional realiza a vinculação deste CID a outro procedimento da tabela de pagamento do SUS (como uma curetagem, por exemplo) na guia de Autorização de Internação Hospitalar (AIH). Embora o CID O04 seja a única forma de identificar a realização do aborto no SIH, não existe uma norma técnica ou uma padronização nacional que oriente sobre a necessidade desse registro e como deve ser realizado. Tal situação leva ao sub-registro, uma vez que o código de procedimento é obrigatório para o serviço receber o pagamento pelo atendimento realizado, enquanto o CID é uma informação complementar.

Sendo o aborto uma questão de grande relevância para a garantia dos direitos sexuais e reprodutivos das mulheres, destaca-se a importância de dados acurados e acessíveis que possam contribuir para a avaliação de necessidades e para o monitoramento de ações voltadas para a implantação de programas que almejem garantir esse direito. Assim, esta pesquisa teve como objetivo analisar os registros hospitalares de atendimentos a mulheres que realizaram aborto legal no Município de Porto Alegre no período de 2013 a 2018, cruzando-os com os dados disponíveis no SIH do SUS. Desta forma, além de realizar um levantamento da diferença quantitativa dos registros realizados nos serviços e os encontrados no sistema de informação do SUS, buscou-se identificar fatores associados à presença do registro no SIH. 


\section{Metodologia}

Foi realizado um estudo transversal com coleta de dados realizada em duas etapas. Na primeira, consultou-se in loco os prontuários de mulheres que realizaram aborto legal entre 2013 e 2018, nos quatro serviços de referência de Porto Alegre. Cada instituição, ao ser solicitada pela equipe de pesquisa, forneceu uma listagem com todos os casos identificados no período. Após, foi aplicada a técnica de linkage entre os dados obtidos nos serviços e aqueles disponíveis no SIH. A partir do resultado da linkage, detectou-se que, entre os casos, havia aqueles que constavam somente nos prontuários hospitalares, aqueles identificados apenas no SIH e os casos que estavam registrados em ambos. A seguir, identificados os casos que estavam registrados somente no $\mathrm{SIH}$, voltou-se aos serviços, em uma segunda etapa de coleta, para busca in loco desses prontuários, com a intenção de chegar o mais próximo possível de um valor fidedigno de prevalência de casos (Figura 1). Ao final, a classificação foi feita em dois tipos: os casos que constavam somente nos prontuários e aqueles que tinham registro tanto nos prontuários como no SIH.

Para a coleta dos dados nos prontuários hospitalares foi criado um instrumento, incluindo as seguintes informações de cada atendimento: nome do hospital, local de residência da mulher atendida, número do cartão nacional do SUS, data da solicitação da AIH, código CID dos registros internos,

\section{Figura 1}

Etapas da coleta de dados e números de registros encontrados e incluídos na pesquisa.

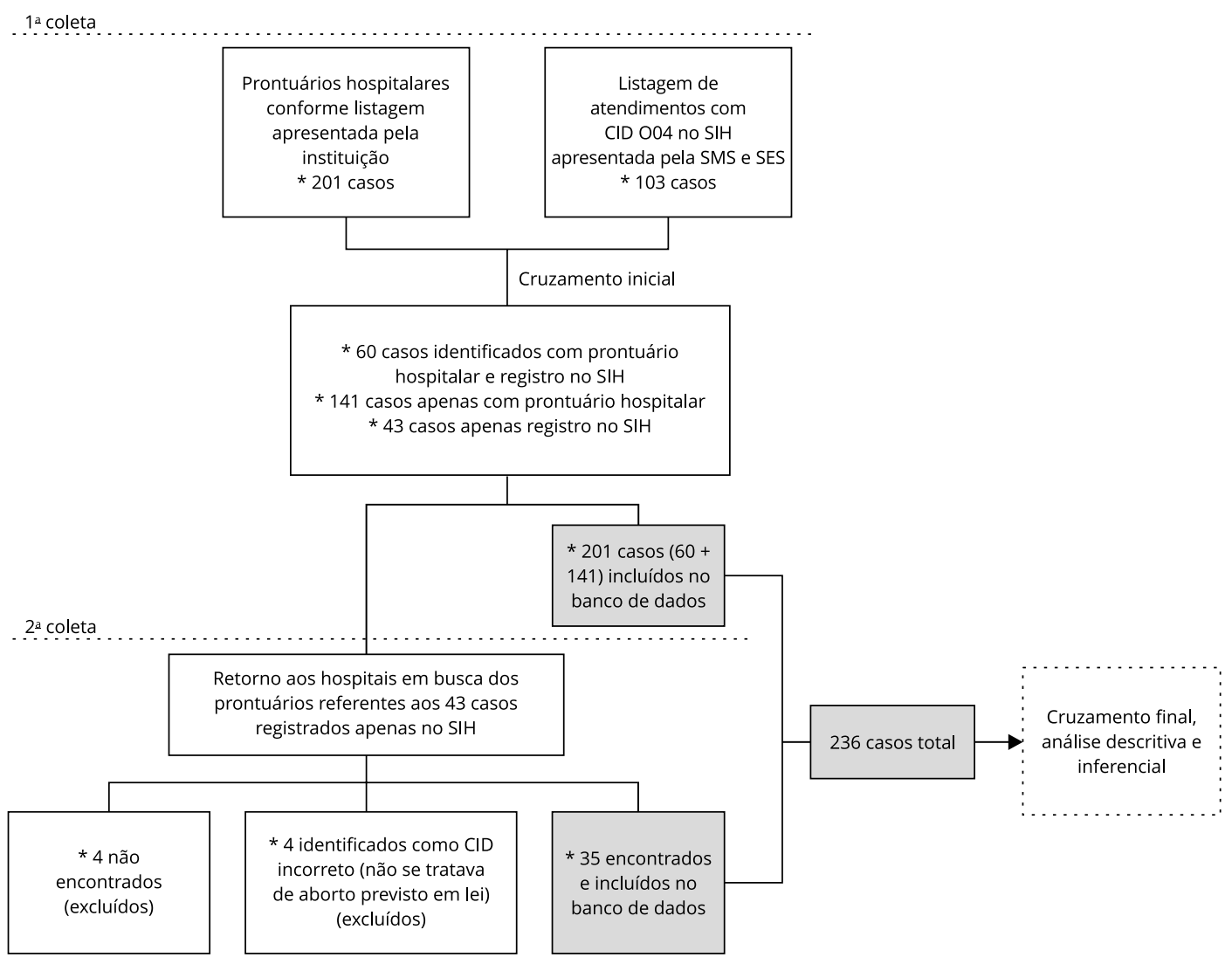

CID: Classificação Internacional de Doenças; SES: Secretaria Estadual de Saúde; SIH: Sistema de Informações Hospitalares;

SMS: Secretaria Municipal de Saúde. 
procedimento registrado na $\mathrm{AIH}$, data da alta e causa prevista em lei para o aborto. Para ter acesso aos prontuários, foi solicitada a cada hospital uma relação dos casos de aborto legal atendidos no período de 2013 a 2018. No total, foram encontrados 201 prontuários nessa etapa. Em cada serviço, houve processos distintos para obtenção da listagem de casos, conforme descrito a seguir.

Hospital A - apresentava prontuário físico e eletrônico. A lista de registros internos foi apresentada pelo ambulatório de atenção à pessoa em situação de violência sexual. O hospital estava realizando um trabalho de pesquisa concomitante sobre violência sexual, o que facilitou o acesso à lista de atendimentos dos casos de violência. Quanto aos casos de anencefalia, foi possível encontrar, com suporte da equipe de informática da instituição, uma listagem de ecografias com o diagnóstico procurado. Após, foram avaliados os prontuários e excluídos os casos em que a gestação não foi interrompida. Nenhum caso de risco de morte materna foi apresentado pelo serviço.

Hospital B - apresentava somente prontuário eletrônico. A lista de registros internos também foi apresentada pelo ambulatório de atenção à pessoa em situação de violência sexual. A relação de registros continha tanto casos de violência sexual como de risco de morte materna. Para acessar os casos de anencefalia foi necessário entrar em contato com a equipe de medicina fetal, que encaminhou os prontuários solicitados.

Hospitais C e D - fazem parte do mesmo grupo hospitalar e, portanto, foi utilizado o mesmo procedimento de coleta em ambos. Apresentavam somente prontuário eletrônico. A lista de registros internos foi apresentada pelo setor de atenção à pessoa em situação de violência sexual. Os hospitais já tinham realizado um trabalho de pesquisa anterior sobre violência sexual, o que facilitou o acesso à lista de atendimentos dos casos de violência. Quanto aos casos de risco de morte materna e anencefalia foram utilizados os registros que alimentam o SIH, encontrados na segunda etapa da coleta, pois não foram listados inicialmente pela instituição.

Foi obtida, por meio de solicitação às Secretarias Estadual e Municipal de Saúde, a listagem de 103 registros de AIH com CID O04 que foram computados no SIH entre 2013 e 2018 nos quatro estabelecimentos estudados. A lista foi gerada a partir dos seguintes dados: serviço de atendimento, número do Cartão Nacional do SUS, data de solicitação da AIH e data da alta. Utilizando essas quatro variáveis, foi realizado o cruzamento do banco de dados construído com os registros obtidos nos serviços com aquele do SIH do SUS. É importante ressaltar que o SIH, embora não seja um sistema de vigilância em saúde, é a principal fonte de informações para análise e planejamento das ações em saúde envolvendo os serviços hospitalares.

Após o primeiro cruzamento dos dados, foram identificados 43 casos que não constavam na lista inicial apresentada pelos serviços, pois estavam registrados apenas no SIH. Desta forma, esses prontuários adicionais tiveram que ser buscados in loco nos serviços. Após oito exclusões (quatro casos que não foram encontrados e quatro que foram registrados com o CID equivocado), mais 35 casos foram incluídos na pesquisa, totalizando 236 registros para a análise, considerando como desfecho a variável presença de registro no SIH, categorizada de forma dicotômica (sim vs. não).

Concluída a construção do banco de dados, prosseguiu-se com a análise estatística. Os resultados descritivos são apresentados em termos de frequências absoluta e relativa. Na parte inferencial, utilizou-se o modelo de regressão de Poisson com variância robusta para avaliar associações com o desfecho, indicado para estudos transversais e desfecho binário (neste estudo, o registro ou não no $\mathrm{SIH}$ ). Foram consideradas como variáveis independentes: hospital em que ocorreu o atendimento (A/B/C; D), se foi colocado o CID O04 nos registros internos (sim; não), se a mulher atendida era residente de Porto Alegre (sim; não), previsão legal para o aborto (violência sexual; outras causas) e procedimento registrado na AIH (curetagem; AMIU [aspiração manual intrauterina]; aborto legal; indução; parto/ cesárea; outro). A escolha dessas variáveis está baseada na hipótese de que elas possam influenciar no desfecho, mais especificamente como fatores de risco para que aconteça o registro no SIH, e as categorias de referência das variáveis independentes foram determinadas com base na literatura e a partir dos resultados encontrados na análise descritiva. Assim, visando um resultado mais detalhado das associações, modelos incluindo efeito de interação entre as variáveis independentes foram realizados. Todas as análises foram realizadas no software PASW Statistics, versão 18.0 (http://www.spss. com.hk/statistics/).

O projeto foi aprovado pelos Comitês de Ética em Pesquisa das instituições responsáveis (CAAE 3.159.095). Todos os cuidados a fim de garantir a confidencialidade e o anonimato dos dados foram 
tomados pelos pesquisadores (não inserção de informações de identificação das pacientes no banco de dados do estudo e assinatura do Termo de Confidencialidade do Uso dos Dados).

\section{Resultados}

Dos 236 casos identificados, 95 (40,3\%) tinham registro no SIH e 141 (59,7\%), apenas nos prontuários internos dos hospitais. A distribuição do total de atendimentos por hospital ao longo do período estudado pode ser observada na Figura 2.

É possível observar que o serviço A, tido como a principal referência para o atendimento de pessoas em situação de violência sexual no estado, sempre realizou um número maior de procedimentos. No entanto, ao comparar 2013 com 2018, todos os hospitais apresentaram um aumento de casos. A série histórica anterior a 2013 não foi incluída no estudo devido ao baixo volume de registros no SIH (apenas um registro em 2010, quatro em 2011 e seis em 2012). Quanto à forma de registro para cada serviço, é possível observar que o serviço A nomeava menos o CID O04 em seus prontuários, e, ao mesmo tempo, foi o que teve menos registros encontrados no SIH (Tabela 1).

Figura 2

Número de abortos legais anuais por local de atendimento entre 2013 e 2018.

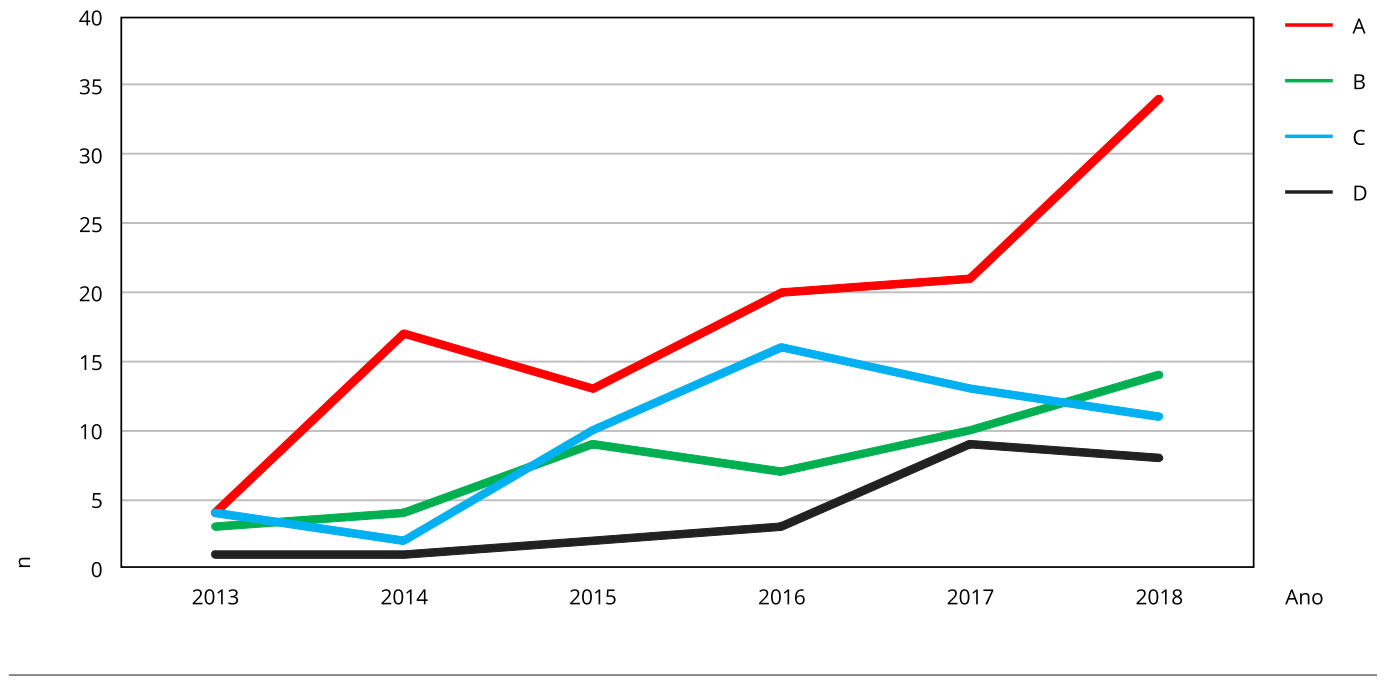

Tabela 1

Percentual, por serviço (A, B, C, D), de registro internos nomeados com CID O04 e de registros presentes no Sistema de Informações Hospitalares (SIH) do Sistema Único de Saúde (SUS).

\begin{tabular}{lcccc}
\hline & A & B & C & D \\
& $\mathbf{n}(\%)$ & $\mathbf{n}(\%)$ & $\mathbf{n}(\%)$ & $\mathbf{n}(\%)$ \\
\hline $\begin{array}{l}\text { CID O04 nomeado no registro interno } \\
\text { Sim }\end{array}$ & $65(59,6)$ & $31(66,0)$ & $44(78,6)$ & $15(62,5)$ \\
$\quad$ Não & $44(40,4)$ & $16(34,0)$ & $12(21,4)$ & $9(37,5)$ \\
Registros no SIH/SUS & & & & \\
$\quad$ Sim & $13(11,9)$ & $33(70,2)$ & $39(69,6)$ & $10(41,7)$ \\
Não & $96(88,1)$ & $14(29,8)$ & $17(30,45)$ & $14(58,3)$ \\
\hline
\end{tabular}

CID: Classificação Internacional de Doenças. 
A análise descritiva de todos os casos pode ser verificada na Tabela 2, que mostra as frequências das variáveis estudadas e a presença de registro no SIH do SUS. É possível observar o número total de abortos realizados nos serviços de Porto Alegre e quantos destes foram registrados no SIH do SUS de acordo com: o hospital que realizou o aborto (A, B, C ou D), se o registro interno do hospital nomeou o aborto como CID O04, se a mulher era residente de Porto Alegre, qual a previsão legal para o aborto (anencefalia, risco de morte materna ou violência sexual) e qual procedimento foi registrado na AIH (curetagem, AMIU, indução, parto/cesárea ou outros).

Ao analisar o total de casos, percebe-se que o Hospital A concentrou o maior número total de atendimentos do município (46,2\%), no entanto, apresenta o menor percentual de registros no SIH (11,9\%). O percentual de registros internos dos hospitais que apresentaram a nomeação do CID O04 foi de $65,7 \%$ e destes, 52,3\% estavam também presentes no SIH, enquanto nos registros sem essa identificação interna no prontuário, apenas 17,3\% foram encontrados no SIH. A violência sexual foi o motivo de previsão legal em $80,9 \%$ dos casos. Apenas seis (2,5\%) abortos estavam relacionados ao risco de morte materna e 16,5\% à anencefalia, porém, diferentemente dos registros relacionados ao risco de morte materna e à anencefalia, a maior parte dos casos de violência sexual não estava no SIH $(63,4 \%)$. Quanto aos tipos de procedimento, os mais frequentes foram a curetagem uterina $(49,8 \%)$ e a AMIU (33,5\%), sendo o registro da AMIU encontrado em maior proporção no SIH. Por fim, ressalta-se que $53,8 \%$ das mulheres atendidas não eram residentes de Porto Alegre, mas não houve diferença quanto à proporção de registros no SIH para esta variável.

Tabela 2

Caracterização dos casos de aborto legal e situação de registro no Sistema de Informações Hospitalares (SIH) do Sistema Único de Saúde (SUS), realizados nos serviços de referência de Porto Alegre, Rio Grande do Sul, Brasil, entre 2013 e 2018.

\begin{tabular}{|c|c|c|}
\hline Variável & $\begin{array}{c}\text { Número de abortos legais realizados } \\
\qquad \mathrm{n}(\%)\end{array}$ & $\begin{array}{l}\text { Número de registros no SIH do SUS } \\
\qquad n(\%) \text { * }\end{array}$ \\
\hline \multicolumn{3}{|l|}{ Serviço } \\
\hline Hospital A & $109(46,2)$ & $13(11,9)$ \\
\hline Hospital B & $47(19,9)$ & $33(70,2)$ \\
\hline Hospital C & $56(23,7)$ & $39(69,6)$ \\
\hline Hospital D & $24(10,2)$ & $10(41,7)$ \\
\hline \multicolumn{3}{|l|}{ CID O04 nomeado no registro interno } \\
\hline Sim & $155(65,7)$ & $81(52,3)$ \\
\hline Não & $81(34,3)$ & $14(17,3)$ \\
\hline \multicolumn{3}{|l|}{ Residentes em Porto Alegre } \\
\hline Sim & $109(46,2)$ & $43(39,4)$ \\
\hline Não & $127(53,8)$ & $52(40,9)$ \\
\hline \multicolumn{3}{|l|}{ Previsão legal para o aborto } \\
\hline Violência sexual & $191(80,9)$ & $70(36,6)$ \\
\hline Risco de morte materna & $6(2,5)$ & $4(66,7)$ \\
\hline Anencefalia ou má formação fetal grave & $39(16,5)$ & $21(53,2)$ \\
\hline \multicolumn{3}{|l|}{ Procedimento descrito na $\mathrm{AlH}$ ** } \\
\hline Curetagem uterina & $116(49,8)$ & $43(37,1)$ \\
\hline AMIU & $78(33,5)$ & $38(48,7)$ \\
\hline Indução & $15(6,3)$ & $7(46,7)$ \\
\hline Parto/Cesárea & $12(5,2)$ & $2(16,7)$ \\
\hline Outros *** & $12(5,2)$ & $2(16,7)$ \\
\hline
\end{tabular}

AIH: Autorização de Internação Hospitalar; AMIU: aspiração manual intrauterina; CID: Classificação Internacional de Doenças.

* Percentual em relação ao número de abortos legais realizados;

** Três prontuários sem o registro da variável;

*** Foram considerados como outros registros de AlH não vinculados diretamente ao aborto (ex.: assistência pós-violência sexual, tratamento em

oncologia ou psiquiatria). 
A Tabela 3 mostra a razão de prevalência (RP) para o desfecho: aborto legal registrado como CID O04 no SIH do SUS.

A prevalência de registro correto do aborto legal no SIH quando existe o CID O04 nos registros internos, é 3,02 (IC95\%: 1,83-4,98) vezes a prevalência de quando este registro não é realizado internamente no hospital. Quanto ao serviço, tendo o Hospital A como referência, a prevalência de registro no SIH foi 5,88 (IC95\%: 3,42-10,13) vezes maior no Hospital B; 5,84 (IC95\%: 3,40-10,00) vezes maior no Hospital C e 3,49 (IC95\%: 1,74-7,00) vezes maior no Hospital D. A escolha do serviço A como categoria de referência na análise de regressão deve-se ao fato do serviço A concentrar o maior número de atendimentos $(46,2 \%)$ e ter a menor proporção de registros no SIH (11,9\%). Para um ajustamento mais refinado do modelo, a interação entre as variáveis "serviço" e "CID O04 nomeado no registro interno" foi incluída, entretanto, esta não se mostrou significativa ( $(\mathrm{p}=0,105)$, o que sustenta a hipótese de que os resultados são independentes, ou seja, aparentemente o efeito do registro interno não depende do serviço que o realiza. Esta análise se justifica porque os serviços possuem uma forma variada de registros. Logo, é necessário elucidar se, por exemplo, o hospital A, ao realizar o registro interno, tem maior chance de ter o registro correto no SIH do que o hospital B. Como o resultado não foi significativo, assumimos que a RP é de 3,02 para todos os hospitais.

Foi encontrada associação significativa com a variável "previsão legal para o aborto": a prevalência de registro no SIH para outras causas foi 1,52 (IC95\%: 1,10-2,09) vezes a prevalência nos casos de violência sexual. No entanto, é importante entender se essa associação difere entre os hospitais. Para

\section{Tabela 3}

Razão de prevalência (RP) bruta de registros de abortos previstos em lei como CID O04 no Sistema de Informação Hospitalar (SIH) do Sistema Único de Saúde (SUS) segundo variáveis independentes.

\begin{tabular}{|c|c|}
\hline Variável & $\begin{array}{c}\text { Casos com registros no SIH } \\
\text { RP (IC95\%) }\end{array}$ \\
\hline \multicolumn{2}{|c|}{ CID O04 nomeado no registro interno } \\
\hline Sim & $3,02(1,83-4,98)$ \\
\hline Não & 1,00 \\
\hline \multicolumn{2}{|l|}{ Serviço } \\
\hline Hospital D & $3,49(1,74-7,00)$ \\
\hline Hospital C & $5,84(3,40-10,00)$ \\
\hline Hospital B & $5,88(3,42-10,13)$ \\
\hline Hospital A & 1,00 \\
\hline \multicolumn{2}{|c|}{ Previsão legal para o aborto } \\
\hline Outras causas * & $1,52(1,10-2,09)$ \\
\hline Violência sexual & 1,00 \\
\hline \multicolumn{2}{|c|}{ Residente de Porto Alegre } \\
\hline Não & $1,04(0,76-1,42)$ \\
\hline Sim & 1,00 \\
\hline \multicolumn{2}{|c|}{ Procedimento descrito na $\mathrm{AlH}$ ** } \\
\hline Curetagem uterina & 1,00 \\
\hline AMIU & $1,31(0,95-1,82)$ \\
\hline Indução & $1,26(0,70-2,27)$ \\
\hline Parto/Cesárea & $0,45(0,12-1,63)$ \\
\hline Outros *** & $0,45(0,12-1,63)$ \\
\hline
\end{tabular}

AlH: Autorização de Internação Hospitalar; AMIU: aspiração manual intrauterina; CID: Classificação Internacional de Doenças; IC95\%: intervalo de 95\% de confiança.

* Refere-se ao agrupamento dos grupos anencefalia e risco de morte materna a fim de possibilitar a análise estatística;

** Três prontuários sem o registro da variável;

*** Foram considerados como outros registros de AlH não vinculados diretamente ao aborto (ex.: assistência pós-

violência sexual, tratamento em oncologia ou psiquiatria). 
explorar melhor essa associação, foi incluída no modelo a interação entre o serviço e a previsão legal para o aborto e esta se mostrou significativa $(\mathrm{p}=0,026)$, indicando que nos serviços $\mathrm{C} / \mathrm{D}$ a prevalência de registro correto no SIH para outras causas é 1,77 (IC95\%: 1,41-2,14) vezes a prevalência para violência sexual, enquanto nos serviços A e B este efeito é nulo. Contudo, tal fato pode ser um viés da coleta, pois foram os dois serviços que apresentaram a listagem de abortos por anencefalia e risco de morte materna, já retirados do SIH.

Por fim, as variáveis "residente em Porto Alegre" e "procedimento descrito na AIH", conforme o apresentado na Tabela 3, não demonstraram associação significativa com o registro correto de aborto previsto em lei no SIH. Desta forma, a descrição do local de residência da mulher e do procedimento utilizado para realizar o aborto não parecem influenciar para que registros de aborto legal sejam mais bem armazenados no SIH do SUS.

\section{Discussão}

A partir da análise inferencial realizada neste estudo, observa-se que a prevalência de registros no SIH é diferente dependendo do serviço que realiza o aborto; mas que, independentemente do hospital, o fato do registro interno descrever o CID O04 aumenta a prevalência de registros no SIH. O aborto justificado pela violência sexual, aparentemente, possui uma prevalência menor nos registros do SUS, contudo, tal resultado foi dependente do serviço, pois apenas dois hospitais apresentaram essa associação. Já o tipo de procedimento e o local de residência não contribuíram para o aumento ou diminuição do registro de aborto legal no SIH.

A dificuldade para a obtenção dos dados desta pesquisa é por si só um importante achado, pois evidencia a falta de padronização e a consequente invisibilização dos abortos previstos por lei realizados nos serviços de referência. Com o processo de coleta, foi possível perceber que nenhum dos serviços apresentava um sistema de registro interno que identificasse com precisão os três casos de aborto previstos em lei. Foram necessárias diferentes abordagens e duas etapas para completar a coleta de dados. A dificuldade de acesso aos dados dos serviços também foi apontada por Madeiro \& Diniz 6 que, em alguns casos, obtiveram os dados para coleta somente após consulta via Lei de Acesso à Informação. Esses obstáculos podem estar relacionados ao contexto de criminalização do aborto e sua repercussão na saúde pública, assim como, todos os aspectos sociais, culturais, morais, bioéticos, religiosos e ideológicos que o envolvem 10. Ademais, o tabu em torno da temática do aborto ainda é fortemente presente na sociedade brasileira, mesmo nas três circunstâncias em que este é permitido por lei 11,12.

Quanto aos dados disponíveis nos sistemas de informação do SUS, é importante destacar que, em nosso país, há diversos sistemas de informação em saúde que armazenam inúmeros bancos de dados 13 . No caso do aborto legal, dois sistemas poderiam ser utilizados para registrar o número de casos: o Sistema de Informações sobre Agravos de Notificação (SINAN), que se refere apenas às situações de violência sexual, e o SIH, em que o CID O04 é utilizado para identificar os três casos previstos em lei, sem distinção. Existem estudos 14 que utilizam os sistemas de informação como fonte oficial para analisar o cenário do aborto no Brasil, contudo, nas revisões sistemáticas 15,16, não encontramos pesquisas que quantificassem os sub-registros.

Os resultados do presente estudo demonstram a dimensão do sub-registro dos abortos legais realizados no Município de Porto Alegre, onde apenas 40,3\% dos atendimentos foram registrados no SIH. Esse número reduzido de registros oficiais não reflete a realidade das mulheres e dos serviços, e pode gerar erros de avaliação e interpretação quanto às necessidades da população em relação ao aborto legal. Além disso, no âmbito mundial, a Organização Mundial da Saúde (OMS) apresenta, em seu documento Abortamento Seguro: Orientação Técnica e de Políticas para Sistemas de Saúde 17, diretrizes para planejamento e gestão da atenção ao abortamento seguro. Nele, aponta o monitoramento dos serviços e dos atendimentos como eixo fundamental para a organização dos sistemas e garantia de direitos sexuais e reprodutivos. Afirma, ainda, que o monitoramento dos indicadores nacionais de abortamento seguro é importante e tem sido amplamente negligenciado.

Nossos dados apontam ainda que, para além do sub-registro, há um número abaixo do esperado de abortos realizados nas condições permitidas pela lei. Segundo o Anuário Brasileiro de Segurança Pública, houve o registro de 271 estupros em 2017 na capital, porém, estudos mostram que as ocorrências 
legais correspondem a apenas $10 \%$ a $30 \%$ dos casos 18,19. Estima-se, também, que pelo menos $5 \%$ das mulheres vítimas de estupro possam engravidar 19. Considerando um percentual de ocorrência de 20\%, Porto Alegre teria em 2017 uma estimativa de 1.355 estupros, com aproximadamente 68 gestações por ano. Todavia, encontramos 191 abortos em casos de violência sexual ao longo de seis anos, o que geraria uma média de 31,8 abortos legais por ano, mas ainda temos que considerar que apenas 46,2\% das participantes residiam em Porto Alegre. Desta forma, chegaríamos a um valor médio de aproximadamente 14,7 atendimentos por ano para mulheres de Porto Alegre.

Outro achado que chama a atenção, merecendo análise mais aprofundada em outras pesquisas, é o registro ínfimo de abortos relacionados ao risco de morte materna. Os seis casos encontrados $(2,5 \%)$ neste estudo ultrapassam o número apresentado por Madeiro \& Diniz 6, que encontraram apenas $1 \%$ ao avaliarem 1.283 prontuários entre 2013 e 2015. Todavia, se considerarmos que este trabalho utilizou um período de análise entre 2013 e 2018, teríamos aproximadamente um caso por ano. Essa escassez de registros também dificultou a análise estatística deste estudo, impossibilitando a discriminação dos casos de risco de morte materna e de anencefalia na avaliação quanto à justificativa legal para o aborto, classificada em violência sexual e outras. Esta pode ser considerada uma limitação desta pesquisa.

Quanto ao número de abortos por anencefalia, foi possível comparar os resultados com os de Madeiro \& Diniz 6: enquanto os autores apresentaram um percentual de 1\%, o nosso estudo encontrou 16,5\%. Fernández et al. 20 encontraram uma prevalência de 0,68/mil casos/ano de anencefalia por nascimentos e Diniz et al. 21 apontam que 84,9\% das gestantes com feto anencefálico preferiram a interrupção legal da gestação. Considerando que Porto Alegre teve 17.579 nascimentos em 2018, o número estimado de casos de anencefalia seria de aproximadamente 12, e 84,9\% desse valor representa cerca de dez casos. Porém, encontramos 39 registros ao longo dos seis anos (6,5 por ano), incluindo os atendimentos para mulheres não residentes da capital. Esse número maior de casos pode dever-se à concentração destes nos serviços de medicina fetal dos hospitais de Porto Alegre, que são referência para todo estado.

Conforme já citado, este estudo possibilitou identificar, por meio da análise inferencial, as variáveis que podem contribuir para um sistema de registros mais acurado, destacando a importância da padronização dos registros relacionados a esse tipo de atendimento. Mesmo sem um código específico que garanta o registro na forma de procedimento, a padronização dos registros internos dos serviços utilizando o CID O04 poderia alterar significativamente o número de identificações corretas no SIH do SUS. Ou seja, é importante identificar e nomear os casos de aborto legal como aborto, entendendo que o código do CID assume, nesse caso, a função de visibilizar um procedimento que atualmente é invisível nos sistemas de informação. Com isso, evitaríamos os registros de outros CIDs - como, por exemplo, o CID Y05 (Agressão sexual através de força física) ou o O269 (Afecções ligadas à gravidez, não especificadas) - que podem ser utilizados no atendimento, mas que não caracterizam o aborto no SIH.

Os resultados da pesquisa evidenciam ainda a falta de uniformidade na forma como os registros são feitos em cada hospital, reforçando a hipótese de que um trabalho de orientação voltado para a padronização entre os serviços também é necessário. Isto porque, mesmo entendendo que o CID é uma uniformização terminológica que permite padronizar a informação sobre o conhecimento de uma doença específica 22, ainda é necessário o aprimoramento dos processos de registros para maior confiabilidade dos dados 23 . Portanto, entende-se como fundamental a elaboração de uma nota técnica nacional com o objetivo de propor um modelo de preenchimento de prontuários e AIH para os atendimentos de mulheres que realizam aborto legal, tendo o código O04 (Aborto por razões médicas e legais) como CID principal. Podendo, ainda, apresentar os CID Y05 (Agressão sexual através de força física), T74.2 (Abuso sexual) e Q00.0 (Anencefalia) como códigos complementares.

Para Menezes et al. ${ }^{24}$, em análises estatísticas sobre os abortos legais, o desafio é a própria identificação dos casos, que depende do código da CID atribuído na guia de internação hospitalar. Além de possíveis falhas nesse registro, a utilização não padronizada desses códigos pode dificultar a comparabilidade dos dados. Em concordância com a autora, este estudo encontrou um sub-registro de 59,7\% dos casos, quando utilizadas somente as informações do SIH.

É importante ressaltar que o serviço com maior volume de atendimentos também foi aquele que apresentou o menor percentual de registros no SIH, evidenciando a necessidade de padronização, 
uma vez que um único serviço pode impactar significativamente no sub-registro de todo o município. No caso desse serviço, havia registros de aborto identificados apenas com o CID Y05 (Agressão sexual através de força física), desta forma, impossibilitando a diferenciação entre os atendimentos que envolviam o aborto previsto em lei e aqueles voltados à pessoa em situação de violência sexual, sem a realização do aborto legal. Assim, mais uma vez, ressalta-se que identificação correta do aborto previsto em lei conforme o CID preconizado (O04) nos registros internos é importante para que esta informação chegue ao SIH.

A literatura sobre o tema tem evidenciado a falta de informações sobre o aborto provocado, visto a própria ilegalidade da prática 4,14,24. No entanto, nosso estudo evidencia que, mesmo os casos previstos em lei, não possuem registros minimamente precisos. Este dado sinaliza a ausência de uma diretriz mais precisa do Ministério da Saúde no tratamento desta questão. A forma como o aborto, mesmo nos casos em que este tem amparo legal, é percebido pela sociedade é um dos principais obstáculos que se apresentam para a plena garantia deste direito 25,26. A cultura de criminalização do aborto pode ser considerada um possível fator para a não nomeação correta dos casos. Uma hipótese é que pode ser socialmente mais confortável para um profissional registrar que realizou um atendimento de violência sexual do que um aborto. Em nossos resultados a prevalência de registros como CID O04 no SIH foi menor para os atendimentos relacionados à violência sexual. Tais resultados foram associados a dois serviços apenas, não tendo sido possível desvinculá-los de um viés de coleta. Contudo, os dados nos permitem lançar a hipótese de que os abortos por risco de vida da mulher ou anencefalia tenham um melhor registro. Outros estudos são necessários para aprofundar essas questões.

O local de residência da mulher, assim como o procedimento utilizado para fazer o aborto, não foram associados à prevalência dos registros no SIH. Todavia, é possível trazer para a discussão a questão de que a maioria dos atendimentos $(53,8 \%)$ foi realizada para mulheres que não residiam no município. Essa situação pode ser reflexo do baixo número de serviços de referência para a realização do aborto previsto em lei no interior do Estado do Rio Grande do Sul. Outro dado relevante é a predominância da curetagem uterina como principal procedimento (49,8\%), uma vez que a Norma Técnica sobre atenção humanizada ao abortamento indica a priorização da AMIU 2. Porém, para avaliar tal questão de forma qualificada, seria necessário um estudo envolvendo as condutas e indicações clínicas de cada caso. Todavia, outros trabalhos como o realizado por Aquino et al. 27 sobre a satisfação do atendimento das mulheres admitidas por aborto em hospitais públicos em Salvador (Bahia), Recife (Pernambuco) e São Luís (Maranhão), também evidencia que a atenção ao aborto nessas cidades encontra-se distante do que propõem as normas brasileiras e os organismos internacionais.

Por fim, destacamos algumas limitações deste estudo: o processo de coleta em duas etapas e seus possíveis vieses (como os casos de anencefalia dos serviços $\mathrm{C}$ e $\mathrm{D}$, encontrados exclusivamente na segunda etapa) e o baixo número de abortos relacionados ao risco de morte materna. A avaliação qualitativa sobre o entendimento dos profissionais a respeito da importância dos registros, o detalhamento de questões clínicas e a análise aprofundada dos processos de registro de cada serviço ficaram fora do escopo deste estudo.

A despeito das limitações, este estudo possibilita uma nova discussão sobre falta de registros dos casos de aborto em sistemas de informações oficiais que possibilitem análises epidemiológicas. Resultado disso é a invisibilidade do aborto legal no modelo de saúde atual, já que o sub-registro dos dados disponíveis faz com que este problema não apareça como uma necessidade de saúde. O presente estudo revela achados importantes sobre dados invisibilizados no sistema de informação do SUS, explorando alguns fatores possivelmente envolvidos no sub-registro.

Em conclusão, os dados apresentados fornecem evidências que auxiliam no direcionamento de ações, como orientações técnicas para gestores e profissionais da saúde, que tenham como objetivo aprimorar os registros do aborto previsto em lei, assim como aponta direções para novas pesquisas sobre o assunto. Os achados apresentados contribuem para aumentar a visibilidade do aborto legal e para promover uma reflexão crítica sobre a imprecisão e o sub-registro dos dados atuais. 


\section{Colaboradores}

Todos os autores participaram da elaboração e revisão do artigo.

\section{Informações adicionais}

ORCID: Maura Carolina Belomé da Silva (00000003-0225-5018); Daniela Riva Knauth (00000002-8641-0240); Rogério Boff Borges (00000002-2548-1889); Luciana Neves Nunes (00000003-0151-1876); Camila Giugliani (0000-00022652-5214).

\section{Referências}

1. Brasil. Decreto-Lei no 2.848 , de 7 de dezembro de 1940. Diário Oficial da União 1940; 31 dez.

2. Área Técnica de Saúde da Mulher, Secretaria de Atenção à Saúde, Ministério da Saúde. Atenção humanizada ao abortamento: norma técnica. 2a Ed. Brasília: Ministério da Saúde; 2011.

3. Departamento de Ações Programáticas Estratégicas, Secretaria de Atenção à Saúde, Ministério da Saúde. Atenção às mulheres com gestação de anencéfalos: norma técnica. Brasília: Ministério da Saúde; 2014.

4. Diniz D, Medeiros M, Madeiro A. Pesquisa Nacional de Aborto 2016. Ciênc Saúde Colet 2017; 22:653-60.

5. Giugliani C, Ruschel AE, Silva MCB, Maia MN, Oliveira DOPS. O direito ao aborto no Brasil e a implicação da atenção primária à saúde. Rev Bras Med Fam Comunidade 2019; 14:179.

6. Madeiro AP, Diniz D. Serviços de aborto legal no Brasil: um estudo nacional. Ciênc Saúde Colet 2016; 21:563-72.

7. Departamento de Regulação, Avaliação e Controle, Secretaria de Atenção à Saúde, Ministério da Saúde. Manual técnico do Sistema de Informação Hospitalar. Brasília: Ministério da Saúde; 2007.

8. Ministério da Saúde. Portaria no 415/SAS/MS, de 21 de maio de 2014. Inclui o procedimento interrupção da gestação/antecipação terapêutica do parto previstas em lei e todos os seus atributos na Tabela de Procedimentos, Medicamentos, Órteses/Próteses e Materiais Especiais do SUS. Diário Oficial da União 2014; 22 mai.

9. Ministério da Saúde. Portaria no 437/SAS/MS, de 29 de maio de 2014. Revoga as Portarias no 224/SAS/MS, de 26 de março de 2014, 272/ SAS/MS, de 2 de abril de 2014, 227/SAS/MS, de 4 de abril de 2014 e 415/SAS/MS, de 21 de maio de 2014. Diário Oficial da União 2014; 30 mai.

10. Santos VC, Anjos KF, Souzas R, Eugênio BG. Criminalização do aborto no Brasil e implicações à saúde pública. Rev Bioét 2013; 21:494508.

11. Patuzzi GC, Giugliani C, Ruschel AE, Silva MCB. Fórum Aborto Legal do RS: relato de experiência de uma estratégia para a garantia dos direitos das mulheres. Caderno de Gênero e Diversidade 2019; 5:151-64.

12. McCallum C, Menezes G, Reis AP. O dilema de uma prática: experiências de aborto em uma maternidade pública de Salvador, Bahia. Hist Ciênc Saúde-Manguinhos 2016; 23:37-56.

13. Ministério da Saúde. A experiência brasileira em sistemas de informação em saúde. Brasília: Ministério da Saúde; 2009.

14. Cardoso BB, Vieira FM, Saraceni V. Aborto no Brasil: o que dizem os dados oficiais? Cad Saúde Pública 2020; 36 Suppl 1:e00188718. 
15. Fonseca SC, Domingues RMSM, Leal MC, Aquino EML, Menezes GMS. Aborto legal no Brasil: revisão sistemática da produção científica, 2008-2018. Cad Saúde Pública 2020; 36 Suppl 1:e00189718.

16. Anjos KF, Santos VC, Souzas R, Eugênio BG. Aborto e saúde pública no Brasil: reflexões sob a perspectiva dos direitos humanos. Saúde Debate 2013; 37:504-15.

17. Organização Mundial da Saúde. Abortamento seguro: orientação técnica e de políticas para sistemas de saúde. 2a Ed. Genebra: Organização Mundial da Saúde; 2013.

18. Fórum Brasileiro de Segurança Pública. Anuário brasileiro de segurança pública. Edição especial 2018. São Paulo: Fórum Brasileiro de Segurança Pública; 2018.

19. Cerqueira D, Coelho D. Estupro no Brasil: uma radiografia segundo os dados da saúde. Brasília: Instituto de Pesquisa Econômica Aplicada; 2014.

20. Fernández RR, Larentis DZ, Fontana T, Jaeger GP, Moreira PB, Garcias GL, et al. Anencefalia: um estudo epidemiológico de treze anos na cidade de Pelotas. Ciênc Saúde Colet 2005; 10:185-90.

21. Diniz D, Penalva J, Fagundes A, Rosas C. A magnitude do aborto por anencefalia: um estudo com médicos. Ciênc Saúde Colet 2009; $14: 1619-24$
22. Laurenti R. Análise da informação em saúde: 1893-1993, cem anos de classificação internacional de doenças. Rev Saúde Pública 1991; 25:407-17.

23. Cesar CLG, Laurenti R, Buchala CM, Figueiredo GM, Carvalho WO, Caratin CV. Uso da Classificação Internacional de Doenças em inquéritos de saúde. Rev Bras Epidemiol 2001; 4:120-9.

24. Menezes GMS, Aquino EML, Fonseca SC, Domingues RMSM. Aborto e saúde no Brasil: desafios para a pesquisa sobre o tema em um contexto de ilegalidade. Cad Saúde Pública 2020; 36 Suppl 1:e00197918.

25. Breilh J. Epidemiologia crítica: ciência emancipadora e interculturalidade. Rio de Janeiro: Editora Fiocruz; 2006.

26. Diniz D, Canabarro V, Mastrella M, Madeiro AP. A verdade do estupro nos serviços de aborto legal no Brasil. Rev Bioét 2014; 22:291-8.

27. Aquino EML, Menezes G, Barreto TV, Alves MT, Alves SV, Almeida MCC, et al. Qualidade da atenção ao aborto no Sistema Único de Saúde do Nordeste brasileiro: o que dizem as mulheres? Ciênc Saúde Colet; 17:1765-76. 
Abstract

Abortion as allowed by law in Brazil is a right that is rarely enforced, due to the multiple barriers to access. Another troublesome issue related to the health system's organization is the difficulty in obtaining reliable and easily accessible records to back monitoring and evaluation of legally authorized abortion. The current study thus aimed to analyze the patient records of women that had undergone legal abortion in the city of Porto Alegre, Rio Grande do Sul State, from 2013 to 2018 and to identify the procedure's prevalence in the Hospital Information System (SIH). The study analyzed on site the patient files for legally permitted abortions performed in the four referral services in the city. The authors cross-analyzed the data with the database of the SIH under the Brazilian Unified National Health System (SUS). Prevalence ratio was estimated with a Poisson regression model with robust variance. Examination of the patient files identified 236 cases of legal abortion, of which 95 (40.3\%) were recorded in the SIH. The cases in which the patient files contained ICD O04 (medical abortion) identified in their internal records had 3.02 times higher prevalence (95\% CI: 1.83-4.98) of being recorded in the SIH than those without the ICD code. The number of records of legal abortions in the SIH differed according to the hospital. However, independently of the hospital, the fact that the internal record listed ICD 004 increased the prevalence of records in the SIH. In conclusion, the lack of standardization and underrecording hindered the collection of trustworthy information in the national database of the SUS, further increasing the invisibility of legal abortion in Brazil.

Legal Abortion; International Classification of Diseases; Information Systems; Records

\section{Resumen}

El aborto está permitido por ley en Brasil, aunque es un derecho poco garantizado, debido a múltiples barreras de acceso. La dificultad de conseguir registros confiables y de fácil acceso, que apoyen acciones de supervisión y evaluación pertinentes para esta temática, es otra cuestión preocupante relacionada con la organización del sistema de salud. De este modo, el objetivo de esta investigación fue analizar los registros de atención a mujeres, que abortaron legalmente en el Municipio de Porto Alegre, Rio Grande do Sul, durante el periodo de 2013 a 2018 e identificar su prevalencia en el Sistema de Informaciones Hospitalarias (SIH). Para tal fin, se evaluaron in loco los historiales de los casos de aborto previstos en ley, efectuados en cuatro servicios de referencia. Los datos obtenidos se cruzaron con el banco de datos del SIH del Sistema Único de Salud (SUS). La razón de prevalencia se estimó mediante el modelo de regresión Poisson con variancia robusta. Se identificaron en los registros médicos, 236 casos de aborto legal, de los cuales 95 $(40,3 \%)$ estaban registrados en el SIH. Los casos cuyos historiales tenían el CIE O04 (aborto por razones médicas), identificado en sus registros internos, contaron con una prevalencia de 3,02 veces (IC95\%: 1,83-4,98) respecto a constar en el SIH, en relación con aquellos que no se identificaron. Se observó que el número de registros de aborto legal en el SIH difería dependiendo del hospital; no obstante, se verificó que, independientemente del hospital, el hecho de que el registro interno describa el CIE O04 aumenta la prevalencia de registros en el SIH. Se concluye que la falta de estandarización y el subregistro dificultan la obtención de información fidedigna en la base de datos nacionales del SUS, aumentando la invisibilidad del aborto legal.

Aborto Legal; Clasificación Internacional de Enfermedades; Sistemas de Información; Registros

Recebido em 27/Set/2020

Versão final reapresentada em 05/Fev/2021

Aprovado em 19/Mar/2021 